\title{
Spin-polarized Current Switching of Co/Cu/Py Pac-man type II Spin-valve
}

\author{
Andrew Lyle', Yang-Ki Hong ${ }^{1 *}$, Byoung-Chul Choi ${ }^{2}$, Gavin Abo' ${ }^{1}$, Seok Bae', \\ Jeevan Jalli' ${ }^{1}$, Jae-jin Lee ${ }^{1}$, Mun-Hyoun Park ${ }^{3}$, and Ryan Syslo' \\ ${ }^{1}$ Department of Electrical and Computer Engineering and MINT Center, The University of Alabama, Tuscaloosa, AL 35487, USA \\ ${ }^{2}$ Department of Physics and Astronomy, University of Victoria, Victoria V8W 3P6, Canada \\ ${ }^{3}$ Hitachi Global Storage Technologies, San Jose, CA 95119, USA
}

(Received 22 June 2010, Received in final form 1 August 2010, Accepted 9 August 2010)

\begin{abstract}
We investigated spin-polarized current switching of Pac-man type II (PM-II) nanoelements in Pac-man shaped nanoscale spin-valves $(\mathrm{Co} / \mathrm{Cu} / \mathrm{Py})$ using micromagnetic simulations. The effects of slot angle and antiferromagnetic (AFM) layer were simulated to obtain optimum switching in less than 2 ns. At a critical slot angle of $105^{\circ}$, the lowest current density for anti-parallel to parallel (AP-P) switching was observed due to no vortex or antivortex formation during the magnetic reversal process. All other slot angles for AP-P formed a vortex or antivortex during the magnetization reversal process. Additionally, a vortex or anti-vortex formed for all slot angles for parallel to anti-parallel (P-AP) switching. The addition of an AFM layer caused the current density to decrease significantly for AP-P and P-AP at slot angles less than $90^{\circ}$. However, at slot angles greater than $90^{\circ}$, the current density tended to decrease by less amounts or actually increased slightly as shape anisotropy became more dominant. This allowed ultra-fast switching with 5.05 and $5.65 \times 10^{8} \mathrm{~A} / \mathrm{cm}^{2}$ current densities for AP-P and P-AP, respectively, at a slot angle of $105^{\circ}$.
\end{abstract}

Keywords ; MRAM, micromagnetic simulation, pac-man, spin-polarized current

\section{Introduction}

Applications for magnetic random access memory (MRAM) require high density and high speed magnetic elements. For MRAM application, the main focus has been on linear magnetic elements such as ellipses [1,2]. A problem with linear magnetic elements is that they have a limited areal density due to inter-element interactions as a result of stray magnetic fields $[3,4]$. In order to increase the areal density of MRAM, closed and semiclosed flux configurations such as vortex and C-shape (Pac-man: PM), respectively, have been intensively studied [5-10]. We previously reported that PM, specifically Pacman type II (PM-II), showed limited element interaction that would allow high areal density MRAM and also that magnetization reversal was completed within 2 ns [6-9]. The different types of Pac-man elements are defined in our previous work [7]. The PM-II is a circle cut with two slot lines to an imaginary inner circle.

Magnetic field switching has traditionally been used to

*Corresponding author: Tel: $+1+205-348-7268$

Fax: +1-205-348-6959, e-mail: ykhong@eng.ua.edu write information into MRAM. Recently, spin-polarized current switching has drawn attention for MRAM due to the simpler and more reliable structure, and low energy consumption [10-13]. Studies for PM shape have focused on magnetic field switching of single layer elements [69]. However, MRAM requires a spin-valve (giant magnetic resistance: GMR) or magnetic tunnel junction (tunneling magnetic resistance: TMR) structure and preferably with spin-polarized current switching. We previously reported simulation results for spin-polarized current switching of elongated Pac-man (EPM) $\mathrm{Co} / \mathrm{Cu} / \mathrm{Ni}_{80} \mathrm{Fe}_{20}$ (Py) [14]. In this paper, we report detailed micromagnetic simulations of spin-polarized current switching of Pacman type II (PM-II) $\mathrm{Co} / \mathrm{Cu} / \mathrm{Py}$ spin-valve. The slot angle and effect of AFM layer are optimized for ultrafast switching.

\section{Simulation}

Fig. 1 shows a schematic of the simulated PM-II spinvalve structure with slot angle of $105^{\circ}$. For all of the simulations, the layers were set to $\mathrm{Co}(20 \mathrm{~nm}) / \mathrm{Cu}(4 \mathrm{~nm}) /$ $\mathrm{Py}(4 \mathrm{~nm})$ for the fixed, spacer, and free layer, respective- 


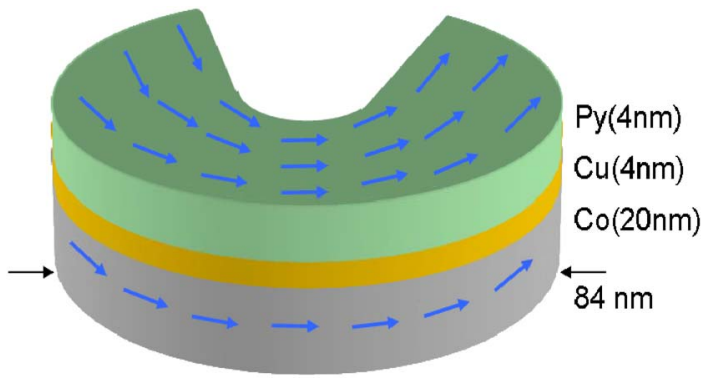

Fig. 1. Schematic of a simulated PM-II $105^{\circ}$ spin-valve structure.

ly. The major axis for the PM-II elements was fixed at 84 $\mathrm{nm}$. Since, the spacer layer thickness is less than the spin diffusion length; the depolarization of the spin-polarized current due to spin-flip scattering can be ignored. The micromagnetic simulations were performed with a code based on the Landau-Lifshitz (LL) equation [15]:

$$
\begin{aligned}
d \vec{M} / d t= & -\left|\gamma /\left(1+\alpha^{2}\right)\right|\left(\vec{M} \times \vec{H}_{e f f}\right) \\
& -\left|(\alpha \gamma) /\left[M_{s}\left(1+\alpha^{2}\right)\right]\right|\left(\vec{M} \times\left(\vec{M} \times \vec{H}_{e f f}\right)\right),
\end{aligned}
$$

where $\gamma$ is the gyromagnetic ratio, and $\alpha$ is a phenomenological damping constant. $M_{\mathrm{s}}$ is the saturation magnetization, and $H_{\text {eff }}$ is the total effective field acting on the magnetization $M$, which mainly includes the applied external field, the exchange interaction, and the demagnetizing field.

The spin transfer torque effect is taken into account by including the Slonczewski term [15]:

$$
\begin{aligned}
d \vec{m}_{1,2} / d t= & {\left[\left(\gamma h J g_{1,2}\right) /\left(2 \pi e \Delta_{1,2} M_{1,2}\right)\right] } \\
& {\left[\vec{m}_{1,2} \times\left(\vec{m}_{1} \times \vec{m}_{2}\right)\right], }
\end{aligned}
$$

where the magnetizations, $m_{1}$ and $m_{2}$, are sequential along positive $z$ direction, $J$ is current density, $h$ is Planck's constant, $e$ is electronic charge, $\Delta$ is the thickness of the layer adjacent to the interface, and the function $g$, which is a function of spin polarizations $\left(\eta_{1}, \eta_{2}\right)$, is defined by Slonczewski [16].

The function $g$ is given by equation [15]:

$$
g_{1,2}=\frac{4 \eta_{1} \eta_{2}^{1 / 2}}{\left(1+\eta_{1}\right)^{2}\left(1+\eta_{2}\right)\left(3+\vec{m}_{1} \cdot \vec{m}_{2}\right)-16 \eta_{1} \eta_{2}^{1 / 2}}
$$

In the simulation, the element is divided with $1 \mathrm{~nm} \times 1$ $\mathrm{nm} \times 1 \mathrm{~nm}$ cubic cell size. Predictor corrector time integration with damping constant $(\alpha)$ of 0.01 and time step of $4.00 \times 10^{-2}$ ps were used in the simulation. The Oersted field created by the spin polarized current pulse is taken into account by the simulator. Other parameters used for the simulation are $\eta=0.26(0.40)$ for $\mathrm{Py}(\mathrm{Co})[10], M_{\mathrm{s}}=$
800 (1414) $\mathrm{emu} / \mathrm{cm}^{3}$ for $\mathrm{Py}(\mathrm{Co})$, exchange constant $\mathrm{A}=$ $1.05 \times 10^{-6}\left(3.05 \times 10^{-6}\right) \mathrm{erg} / \mathrm{cm}$ for $\mathrm{Py}(\mathrm{Co})$, and zero magnetic anisotropy constant [15]. The magnetostatic fields between the free and fixed layer were taken into account in the simulations [15]. For each PM-II slot angle, an initial simulation was started in the demagnetization state and the anisotropy and demagnetization energy were minimized when no field or current is applied to reach its equilibrium state (i.e. remanent state). The remanent state was used as the initial magnetization configuration for the spin-polarized current switching simulation for each slot angle.

The effect of slot angle and the addition of an antiferromagnetic (AFM) layer were studied to determine the optimized PM-II spin-valve structure. The angle was varied from $45^{\circ}$ to $180^{\circ}$ in $15^{\circ}$ or $30^{\circ}$ increments. The small step size of $15^{\circ}$ was used near $105^{\circ}$ where optimum switching was observed. An AFM layer was added to the PM-II elements to determine the effect on the magnetization reversal. An AFM layer was added to the simulation by applying a 600 Oe pinning field to the fixed layer along the major axis [17]. It is assumed that the AFM layer will have little effect on the free layer, so no pinning field was applied to the free layer [13].

\section{Results and Discussion}

Fig. 2(a) shows the current density for anti-parallel to parallel (AP-P) switching at various slot angles for PM-II elements with and without AFM layer. For AP-P switching, the applied current flows from the free layer to the fixed layer with a 200 ps pulse width (as shown in the inset of Fig 2(a)). The AP-P switching for PM-II elements with and without the AFM layer shows a similar trend. A minimum current density of 5.0 and $5.3 \times 10^{8} \mathrm{~A} / \mathrm{cm}^{2}$ is observed at a slot angle of $105^{\circ}$ with and without an AFM layer, respectively.

Fig. 2(b) shows snap-shots of AP-P switching for PM-II element at a slot angle of $105^{\circ}$ without an AFM layer. The first five snapshots show the Py free layer during the switching process, while the last snapshot shows the unchanged fixed layer after the switching process. At 60 ps, the magnetization has rotated clockwise approximately $90^{\circ}$ in the Py free layer. By the end of the current pulse at $200 \mathrm{ps}$, the magnetization has completely rotated to the parallel state. Finally, at $2 \mathrm{~ns}$, it fully relaxes to the parallel configuration and the Co fixed layer remains unchanged.

The addition of an AFM layer alters the current densities for AP-P switching specifically at slot angles of $45^{\circ}$ and $75^{\circ}$. This is partially due to the fact that the AFM 


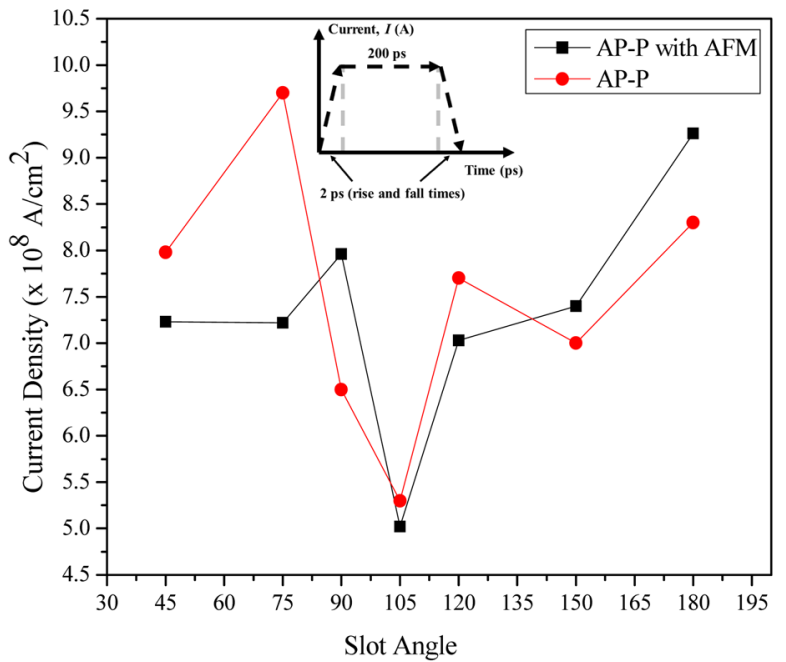

(a)

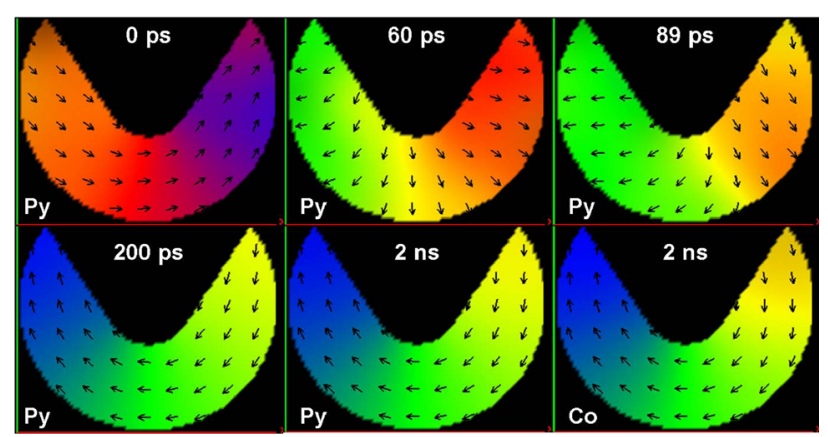

(b)

Fig. 2. (color online) (a) AP-P switching current density as a function of slot angle with and without AFM layer and (b) snap-shots of AP-P for slot angle of $105^{\circ}$ without AFM layer. First five snap-shots of Py layer and last of Co layer. The color code for the plots is Red: $+\mathrm{M}_{\mathrm{x}}$, Green: $-\mathrm{M}_{\mathrm{x}}$, Yellow: $-\mathrm{M}_{\mathrm{y}}$, and Blue: $+\mathrm{M}_{\mathrm{y}}$.

layer prevents the vortices that form during the magnetization reversal process from annihilating. For slot angles greater than $75^{\circ}$, the current densities follow a similar trend with and without an AFM layer. The reason that a slot angle of $105^{\circ}$ shows the lowest current density for AP-P switching without the AFM layer is because no vortex or anti-vortex (defined as having the opposite polarity to the vortex) forms [14]. Vortex anti-vortex pair formation can be caused by short current pulses $[18,19]$. Moreover, a $6 \%$ decrease in current density is observed when the AFM layer is applied at a slot angle of $105^{\circ}$, where an anti-vortex forms and is driven out of the element through one of the tips. The Oersted field, which is proportional to current, plays a significant role in the aforementioned switching process. Without the Oersted field, vortices remain likely since there is not enough energy to remove them (i.e., the Oersted field adds about $4 \times 10^{-11} \mathrm{erg}$ in

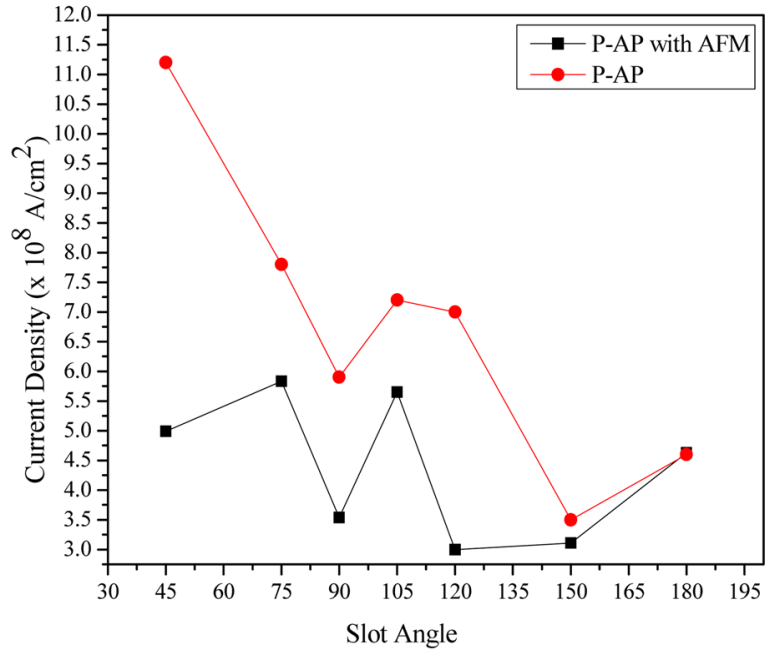

(a)

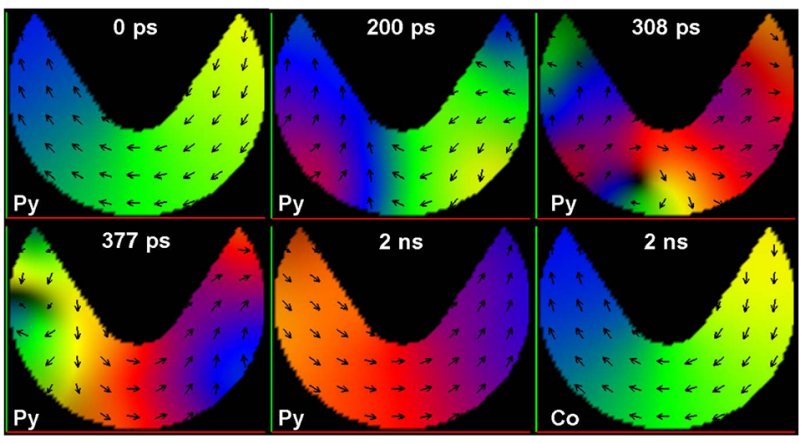

(b)

Fig. 3. (a) P-AP switching current density as a function of slot angle with and without AFM layer and (b) snap-shots of P-AP for slot angle of $105^{\circ}$ with AFM layer. First five snap-shots of Py layer and last of Co layer. The color code for the plots is Red: $+M_{x}$, Green: $-M_{x}$, Yellow: $-M_{y}$, and Blue: $+M_{y}$.

magnitude of external energy during the applied current pulse), and the element does not switch. Thus, the change in switching mechanism caused by the AFM layer does not solely account for the reduction in switching current density.

Fig. 3(a) shows the current density for parallel to antiparallel (P-AP) switching for PM-II element with and without AFM layer. For P-AP switching, the current is applied from fixed layer to the free layer with a 200 ps pulse width. Regardless of AFM layer, a decrease in current density for increasing slot angle is observed for PAP. A vortex or anti-vortex forms for P-AP switching for all slot angles with and without AFM layer. However, the addition of the AFM layer lowered the current density of P-AP switching for all angles except $180^{\circ}$, where the current density remains the same. This may be attributed to the fact that for these angles the AFM layer prevented the annihilation of the vortex during the reversal process. 


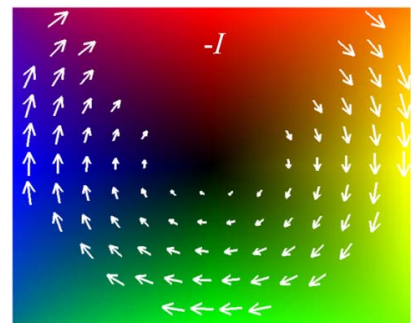

(a)

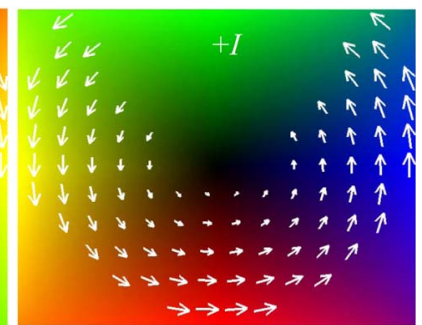

(b)
Fig. 4. Spatial distribution of the current-induced Oersted field in Py layer of $105^{\circ}$ PM-II with AFM for (a) AP-P and (b) PAP switching. The color code for the plots is Red: $+M_{x}$, Green: $-M_{x}$, Yellow: $-M_{y}$, and Blue: $+M_{y}$.

Instead, the vortex was removed from the PM-II element through one of the tips. Consequently, the current density was reduced by $22 \%$ at a slot angle of $105^{\circ}$. This allows the difference in current densities for AP-P and P-AP switching to reduced to $12 \%$ at a slot angle of $105^{\circ}$.

Fig. 3(b) shows snap-shots of P-AP switching for PM-II element with slot angle of $105^{\circ}$ with an AFM layer. At $200 \mathrm{ps}$, the magnetization has reversed near the left edge of the Py element. By 308 ps, a vortex forms at the bottom of the PM-II element. Near 377 ps, the vortex is forced out of the PM-II element through the left tip. Finally, at $2 \mathrm{~ns}$, it has fully relaxed into the anti-parallel configuration, while the Co fixed layer remains unchanged. Similar to AP-P switching of $105^{\circ}$ PM-II with AFM layer, the Oersted field drove the reversal process. The spatial distribution of the current-induced Oersted field conforms to the PM-II shape in the clockwise direction for AP-P and counter-clockwise for P-AP as shown in Fig. 4(a) and (b), respectively. The frequent observation of vortex or anti-vortex formation may originate from small $g_{1,2}$ on the order of 0.1 .

For low slot angles, the AFM layer is effective in lowering the current density substantially for both AP-P and P-AP switchings. This is due to the fact that the fixed and free layer for the PM-II elements without the AFM layer are weakly antiferromagnetically coupled [20]. When the AFM layer is applied, the fixed layer is strongly pinned and the free layer is allowed to precess more freely. As a result, the current density for the soft free layer is reduced similar to results observed with composite free layers and the magnetic reversal process changes [20-23]. At larger slot angles, shape anisotropy seems to play a dominant role in the magnetization process [6,7]. As a result, the AFM layer has an insignificant effect and the current density decreases by a smaller amount or actually increases as observed in AP-P switching.

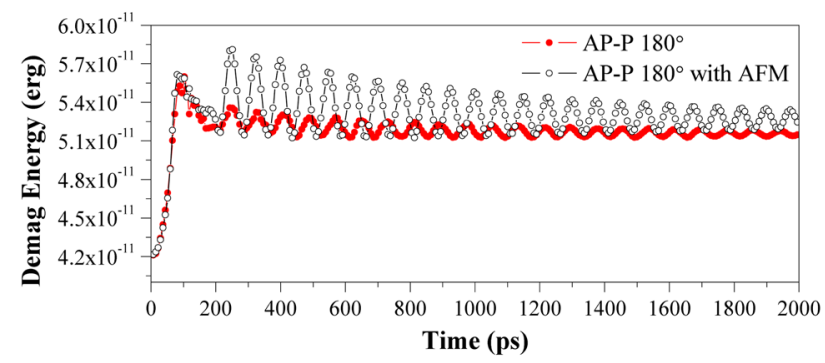

(a)

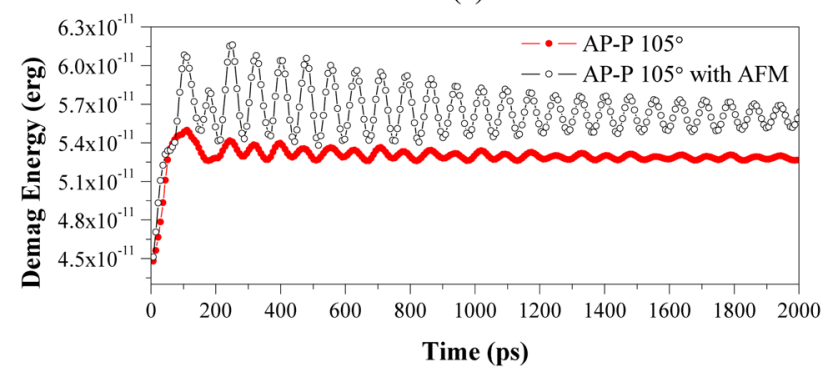

(b)

Fig. 5. Demagnetization energy for AP-P switching with and without AFM layer for slot angle of (a) $180^{\circ}$ and (b) $105^{\circ}$.

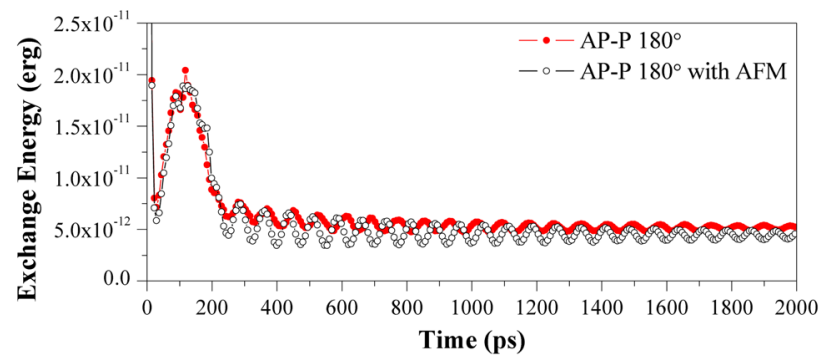

(a)

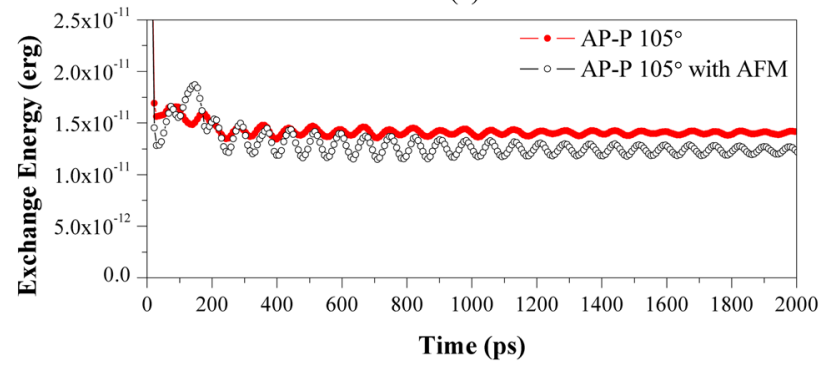

(b)

Fig. 6. Exchange energy for AP-P switching with and without AFM layer for slot angle of (a) $180^{\circ}$ and (b) $105^{\circ}$.

Fig. 5 and 6 show the demagnetization and exchange energy for AP-P switching at selected PM-II slot angles, respectively. At slot angle of $105^{\circ}$, the AFM layer decreases the current density from $5.65 \times 10^{8} \mathrm{~A} / \mathrm{cm}^{2}$ to $5.05 \times 10^{8} \mathrm{~A} / \mathrm{cm}^{2}$ for AP-P switching, while the current density for the slot angle of $180^{\circ}$ increased. The exchange and demagnetization energies are similar for a slot angle of $180^{\circ}$ with and without an AFM when the 200 ps 
current pulse is applied. After the pulse is turned off, the exchange and demagnetization energies follow a similar trend but oscillate with larger amplitude when the AFM layer is applied. The small oscillations in the exchange and demagnetization energy for PM-II without an AFM layer is attributed to the weak coupling between the fixed and free layer through magnetostatic fields damping free layer precession.

For a slot angle of $105^{\circ}$, the demagnetization and exchange energies show different behaviors when an AFM layer is applied, this corresponds to a change in the magnetic reversal process. When an AFM layer is applied, a sharp peak in the exchange and demagnetization energy is observed while the current is applied, this corresponds to the nucleation of a vortex. After the pulse is removed, the AFM layer increases the oscillation amplitude in demagnetization and exchange energy similar to the slot angle of the $180^{\circ}$ case. This indicates that the AFM layer effectively changes the magnetic reversal process and allows the free layer to precess more freely as mentioned previously. The role of the AFM layer could be related to the angular dependence of $g_{1,2}$. Yet, more study is needed to further understand the significance of the AFM pinning.

\section{Conclusion}

Spin-polarized current switching of a PM-II spin-valve structure has been optimized by micromagnetic simulation for ultra-fast switching. Magnetization reversal completed within 2 ns and a difference in current densities of only $12 \%$ to switch AP-P and P-AP for slot angle of $105^{\circ}$ show promising results. These results for spin-polarized current switching in combination with semi-closed-flux configurations make the PM-II magnetic nanoelement an attractive element for MRAM application.

\section{Acknowledgement}

This work was supported in part by the E. A. "Larry" Drummond Endowment at the University of Alabama.

\section{References}

[1] N. C. Emley, F. J. Albert, E. M. Ryan, I. N. Krivorotov, D. C. Ralph, J. M. Daughton, and A. Jander, Appl. Phys. Lett. 84, 4257 (2004).

[2] P. M. Braganca, I. N. Krivorotov, O. Ozaty, A. G. F.
Garcia, N. C. Emley, J. C. Sankey, D. C. Ralph, and R. A. Buhrman, Appl. Phys. Lett. 87, 112507 (2005).

[3] J. Janesky N. D. Rizzo, L. Savtchenko, B. Engel, J. M. Slaughter, and S. Tehrani, IEEE Trans. Magn. 37, 2052 (2001).

[4] S. Tehrani J. M. Slaughter, M. Deherrera, B. N. Engel, N. D. Rizzo, J. Salter, M. Durlam, R. W. Dave, J. Janesky, B. Butcher, K. Smith, and G. Grynkewich, Proc. IEEE 91, 703 (2003).

[5] H. Hu, H. Wang, M. R. McCartney, and D. J. Smith, J. Magn. Magn. Mater. 290, 234 (2005).

[6] M. H. Park, Y. K. Hong, S. H. Gee, D. W. Erickson, and B. C. Choi, Appl. Phys. Lett. 83, 329 (2003).

[7] M. H. Park, Y. K. Hong, S. H. Gee, D. W. Erickson, T. Tanaka, and B. C. Choi, J. Appl. Phys. 95, 7019 (2004).

[8] B. R. Pujada, J. Svendsen, K. O. Chipeniuk, B. C. Choi, M. H. Park, Y. K. Hong, S. H. Gee, and D. W. Erickson, J. Appl. Phys. 96, 4362 (2004).

[9] H. Han, Y. K. Hong, M. H. Park, B. C. Choi, S. H. Gee, J. F. Jabal, G. Abo, A. Lyle, B. Wong, and G. W. Donohoe, IEEE Trans. Magn. 41, 4341, (2005).

[10] B. C. Choi, J. Rudge, E. Girgis, J. Kolthammer, Y. K. Hong, and A. Lyle, Appl. Phys. Lett. 91, 22501 (2007).

[11] H. Meng and J. P. Wang, Appl. Phys. Lett. 89, 152509 (2006).

[12] X. Yao, H. Meng, Y. Zhang, and J. P. Wang, J. Appl. Phys. 103, 07A717 (2008).

[13] G. Finnocchoi, I. N. Krivorotov, L. Torres, R. A. Buhrman, D. C. Ralp, and B. Azzerboni, Phys. Rev. B 76, 174408 (2007).

[14] A. Lyle, Y. K. Hong, B. C. Choi, G. S. Abo, M. H. Park, S. H. Gee, J. Jalli, S. Bae, and G. W. Donohoe, IEEE Trans. Magn. 45, 2367 (2009).

[15] M. R. Scheinfein, LLG Micromagnetic Simulator ${ }^{\mathrm{TM}}$

[16] J. Slonczewski, J. Magn. Magn. Mater. 159, L1 (1996).

[17] L. Wang, J. H. Giusti, and J. Fernando-de-Castro, J. Appl. Phys. 89, 7006 (2001).

[18] Y. Liu, S. Gliga, R. Hertel, and C. M. Schneider, Appl. Phys. Lett. 91, 112501 (2007).

[19] R. Hertel, S. Gliga, M. Fähnle, and C. M. Schneider, arXiv:cond-mat/0611668v2, (November 2006).

[20] S. S. P. Parkin, R. Bhadra, and K. P. Roche, Phys. Rev. Lett. 66, 2152 (1991).

[21] C. T. Yen, W. C. Chen, D. Y. Wang, Y. J. Lee, C. T. Shen, S. Y. Yang, C. H. Tsai, C. C. Hung, K. H. Shen, M. J. Tsai, and M. J. Kao, Appl. Phys. Lett. 93, 092504 (2008).

[22] Y. Jiang, T. Nozaki, S. Abe, T. Ochiai, A. Hirohata, N. Tezuka, and K. Inomata, Nature 3, 361 (2004).

[23] J. P. Wang, W. K. Shen, J. M. Bai, R. H. Victora, J. H. Judy, and W. L. Song, Appl. Phys. Lett. 86, 142504 (2005). 